\title{
BSS: a brokering model for service selection using integrated weighting approach in cloud environment
}

\author{
Sameer Singh Chauhan ${ }^{1}$, Emmanuel S. Pilli ${ }^{{ }^{*}}$ (D) and R. C. Joshi ${ }^{2}$
}

\begin{abstract}
Cloud providers shares their resources and services through collaboration in order to increase resource utilization, profit and quality of services. The offered services with different access patterns, similar characteristics, varied performance levels and cost models create a heterogeneous service environment. It becomes a challenging task for users to decide a suitable service as per their application requirements. Cloud broker, an inter-mediator is required in service management to help both cloud providers and users. Cloud broker has to store all the information related to services and feedback of users on those services in order to provide the best services to end-users. Brokering model for service selection (BSS) has been proposed which employs integrated weighting approach in cloud service selection. Subjective and objective weights of QoS attributes are combined to compute integrated total weight. Subjective weight is obtained from users' feedback on QoS attributes of a cloud service while objective weight is computed from benchmark tested data of cloud services. Users' feedback and preferences given to QoS parameters are employed in subjective weight computation. Objective weight is computed using Shannon's Entropy method. Total weight is obtained by combining subjective and objective weights. BSS method is employed to rank cloud services. Simulation with a case study on real dataset has been done to validate the effectiveness of BSS. The obtained results demonstrate the consistency of model for handling rank reversal problem and provides better execution time than other state-of-the art solutions.
\end{abstract}

Keywords: Cloud computing, Service selection, Cloud broker, Integrated weight

\section{Introduction}

Cloud Computing delivers computing services such as storage, networking, processing, etc. on demand and subscription basis over Internet. These services are offered as Infrastructure as a Service (IaaS), Platform as a Service (PaaS), and Software as a Service (SaaS) [1]. IaaS provides infrastructure facilities to run, deploy and manage virtual machines. SaaS provides complete set of applications such as customer relationship management (CRM). PaaS provides platforms such as Google App Engine to develop applications [2].

\footnotetext{
*Correspondence: espilli.cse@mnit.ac.in

${ }^{1}$ Department of Computer Science and Engineering, Malaviya National

Institute of Technology Jaipur, Rajasthan, India

Full list of author information is available at the end of the article
}

Cloud Computing delivers services on pay-per-use model. Organizations can outsource their computing requirements to a Cloud. They do not require to set up an IT infrastructure and hence can save lots of money. They can purchase required computing facilities from any cloud provider as per their QoS requirements [1]. They don't have to worry about maintenance and management of resources. They can change or upgrade resources as per requirement without worry of devaluation and elimination of resources and technologies [3].

Due to proliferation of cloud services by various cloud providers, service selection became a difficult task to cloud users [4]. Cloud providers offer similar types of services on varying price and performance levels. One cloud provider may offer storage services on cheaper rate while 
computing service on higher price. Amazon EC2 [5] offers its same computing services on different prices. It became a very difficult task for a user to select an appropriate services as per QoS demands. QoS demands include both functional and non-functional parameters [6]. Every cloud provider can not fulfill all QoS requirements. There may be a trade-off between fulfilling different QoS demands by service providers. Users have to search and test all cloud services. This becomes infeasible when number of service providers are more.

Multiple cloud environment [7] provides a platform where service providers can offer their cloud services through collaboration. Cloud computing with multiple cloud providers is the new emerging computing paradigm [8]. A huge pool of services is created with varied services such as computing, data, storage, applications [9] in multiple cloud environment. Many services of the pool are of same type but offered with different access methods and posses diverse characteristics [10]. Users have to identify and select desired service from this pool. It causes difficulty to them for selection of the best one because of similar functionality of services but offered differently. An inter-mediator is required to handle issues of service providers and users. So that users have not to visit each and every cloud provider for services. Cloud broker [11] works as an inter-mediator. Cloud broker collects all the required attributes of services from different service providers and offers discovery, ranking, selection, allocation, etc. of services as per requested QoS demands [12].

The nature of Cloud environment is dynamic, heterogeneous and distributed. Therefore cost and performance based metrics and benchmarks of traditional computing environments such as high performance, grid, and cluster computing etc. can not be applied to cloud computing environment [13]. Many organizations such as Information and Communication Technology Service Quality (ICTSQ), Application Performance Index (APDEX), ISO/IEC 9126, eSourcing Capability Model-Client Organizations (eSCM-CL) and Service Measurement Index (SMI) [14] are trying to frame standards and benchmarks for evaluating different cloud services. Cloud service ranking faces many challenges. One of them is collection of QoS values of services [15]. There are many entities which provides QoS data both functional and non-functional of cloud services. End users provide feedback of a cloud service on various QoS parameters. These values can be in linguistic terms which is to be converted in quantifiable form [16]. Cloud service repositories provide QoS values of cloud services which is difficult to measure. Trusted third party monitoring tools provide live QoS data of cloud services. Another challenge is how to measure time varying values of QoS attributes e.g. virtual machine's performance found varying over time compared with the SLA offered [5]. Rank a cloud service on gathered QoS data is also difficult because these data are not in one format.

The above issues make cloud service selection a complex problem which involves more than one QoS criteria to be addressed. Service selection in cloud environment is a multi criteria decision making (MCDM) [17] problem. QoS attributes may be independent or inter-dependent. The QoS attribute and its sub-attribute affects in cloud service ranking and selection [18]. Users demand various QoS requirements as per their applications' need. The user QoS needs can be categorized in to two category: One which are interested only main QoS attributes such as price. Another which are interested not only main QoS attributes but also in sub-attributes. For example price attribute can be decomposed in inbound bandwidth price, outbound bandwidth price and base plan.

Several researchers have proposed techniques to solve cloud service selection problem using multi-criteria decision making approach. There is a lack of hybrid multicriteria decision making technique which employs both subjective and objective assessment of cloud services attributes. The QoS attributes values play an important role in evaluation of services. The weight value assigned to QoS attribute describes its relative Joyent with comparison to other attributes in the evaluation process. Every QoS attribute cannot be assigned equal weight because of its diversification and meaning in decision making [19]. Many approaches can be found in literature to obtain weight of a QoS attribute [20] which can be classified into subjective and objective. The obtained weight from these approaches is known as subjective weight and objective weight [21].

In this paper, we have proposed a brokering model for service selection in cloud environment which employs integrated weighting approach named as BSS. Integrated weight is computed from subjective and objective assessment of cloud services. Subjective assessment of any cloud service is done using users' feedback on various QoS attributes. The feedback is in linguistic terms which is converted into crisp numerical value by employing fuzzy set theory. BSS employs fuzzification and defuzzification methods to compute crisp value of end users' feedback data. Objective assessment of a cloud service is performed through benchmark tested data. Therefore benchmark tested data are also employed to evaluate cloud services. BSS integrates both subjective and objective assessment of cloud service to find best service. We have extended our previous work [22] in this paper with BSS model. Our previous work do not consider end users' feedback and different weighting approaches in service selection. BSS collects end users' feedback on various QoS attributes, converts into crisp numerical value and employs it for service evaluation. It also gathers benchmark tested data of 
various cloud services for service evaluation. BSS computes integrated Joyent in the form of weight which is applied to rank cloud services as per users QoS demands.

Salient contributions of the paper are:

- A brokering model for service selection in cloud environment.

- A hybrid method which employs integrated weighting technique to compute overall weight value of QoS attributes of a cloud service.

- A cloud service selection method which employs integrated weight of QoS attributes for ranking.

- Extensive simulations to evaluate effectiveness of proposed model. Sensitivity analysis with existing literature has been performed along with other results in evaluation.

The rest of the paper is organized as follows: Related work is discussed in "Related work" section. "Brokering model for service selection (BSS)" section discusses proposed brokering model, BSS. Service selection method is discussed in "BSS technique" section. "Experiment and result" section provides experimental environment and discussion of results. Conclusion and future work is provided in "Conclusion" section.

\section{Related work}

Service selection in multiple cloud environment is one of the most challenging and researched topic for researchers. This section discusses literature related to cloud brokering techniques for cloud service selection. Recent research work in cloud service selection has focused on providing methods and mechanism to rank services which will help users in selecting best service.

Patiniotakis et al. [23] have presented a brokerage service based on preference-based recommender system for ranking cloud services. It applies multi-criteria decision making to optimize the brokerage. The recommender system considers heterogeneous characteristics of service in evaluation. Ranking of services is done with fuzzy AHP [24] method which considers multi-objective assessment of services. It considers precise and imprecise metrics to deal with fuzziness of cloud services opinions. The method provides more expressive and unified way to gather users' preferences.

Chan et al. [25] have proposed an enhanced fuzzy Analytic Hierarchy Process (AHP) which is based on fuzzy AHP. It uses triangular fuzzy number to convert linguistic terms into numeric form. The method finds weight vectors of customer requirements and provides prioritizing them. A case study based evaluation of proposed method has been given which demonstrates the effectiveness of method by capturing vagueness of human judgment in weight calculation.
Garg et al. [26] have proposed SMICloud framework for ranking cloud services using AHP [24] method. Service Measurement Index (SMI) parameters including functional and non-functional QoS parameters are formulated. Main attribute and sub-attributes of QoS parameters are considered for evaluating a cloud service. The rank of cloud services are computed using AHP method.

Patiniotakis et al. [27] have proposed an approach for cloud service evaluation based on heterogeneous service characteristics. It provides alternative classification of metrics used for cloud service ranking. Proposed approach uses method to convert fuzziness of service characteristics into numerical form. Proposed approach provides unified way to assess multi-objective criteria of a cloud service. Proposed model implements fuzzy AHP method to evaluate it.

Sidhu and Singh [28] have proposed a framework based on subjective attributes assessment to compute trust of a cloud service provider. The framework employs AHP [29] and TOPSIS [30] methods to compute trust value of a cloud service. AHP method is employed to compute Joyent in the form of a weight value of QoS parameters. TOPSIS is employed to compute trust value of a cloud service considering weight value provided by AHP method.

Rehman et al. [31] have proposed a multi-criteria decision making method for cloud service selection. The method distinguishes service on its various QoS attributes. Proposed approach has considered services which are similar in various specifications but differ in performance.

Anastasi et al. [32] have proposed a genetic approach for cloud brokering. It finds suitable IaaS services which satisfies QoS requirements of applications. Proposed approach mitigates scalability and vendor lock-in issues by finding near optimal solution according to customer preferences. It supports different cost models of cloud providers.

Sundareswaran et al. [33] have proposed a brokerage architecture for service selection. Large number of cloud service providers and thier information are managed through a indexing technique which is given by architecture. It also contains one method which uses query algorithm for finding suitable service or set of services. It also aggregates services if single service cannot satisfy the requirements.

Qu et al. [34] have proposed a framework for cloud service selection which aggregates subjective assessment for users and objective performance from a trusted third party. The subjective and objective attributes of a cloud service are normalized and aggregated using simple additive weighting system. The framework provides a technique to filter feedback from malicious users. A case study has been proposed to verify the framework. 
Kumar et al. [35] have proposed a framework for selection of cloud services considering fuzzy environment. Users can provide their input for QoS parameters through linguistic terms. AHP and fuzzy TOPSIS methods are employed for selection of cloud services. AHP has been employed to compute weight of QoS parameters. TOPSIS with triangular fuzzy number has been employed to rank cloud services.

Paunovic et al. [36] have proposed a two-stage fuzzy logic model for cloud supplier evaluation and selection. The model is based on Fuzzy Analytic Hierarchy Process (FAHP). It applies fuzzy logic approaches based on technology perspective to model users requirements. The model helps decision makers to extract knowledge from database to find best cloud providers. It is evaluated through a case study based numerical example.

Esposito et al. [16] have proposed a method for storage cloud service selection in cloud environment. It uses fuzzy set theory to overcome problems associated in subjective preference given by users to QoS levels. Dempster-shafer theory of evidence and game theory approach are used to resolve service selection problem and find best service as per users QoS requirements.

Alam et al. [37] have proposed an integrated uncertainty aware hybrid multi criteria decision making model for cloud service evaluation and selection. The model is integration of Fuzzy Analytic Hierarchy Process [38] and Weighted Aggregated Sum Product Assessment method [39]. The model can be used for finding best services of IaaS, SaaS and PaaS types. Proposed model provides rank of services based on QoS attributes of cloud services. Case study based evaluation of the model has been presented.

Tajvidi et al. [40] have proposed a fuzzy logic framework for selection of cloud services. The framework consists of four modules: interface, QoS management, service selection process and cloud service repository. Interface module gathers data from users about their requirements. QoS management module captures linguistic weight of requested criteria. It converts into crisp value through triangular fuzzy numbers. Service selection modules performs two tasks: compute the QoS metrics of services and assign rank to the services. Cloud service repository is responsible to collect different QoS values of services from different sources. Case study based evaluation of proposed framework has been done to validate it.

Rohit and Rakesh [41] have proposed a framework for cloud service selection based on Gaussian TOPSIS (G-TOPSIS) method. G-TOPSIS considers QoS feedback of cloud services provided by different users for ranking them. It also considers priority assigned to QoS parameters by end user. The QoS values are normalized using cumulative Gaussian density function. The framework has been tested on real time dataset obtained from CloudHarmony. G-TOPSIS ranks cloud services similar to TOPSIS method. G-TOPSIS solves rank reversal problem. G-TOPSIS does not consider inter-dependency of QoS attributes as well as qualitative QoS metrics. It does not consider QoS feedback given in linguistic terms.

Hussain et al. [42] have proposed a framework, CSSaaS for cloud service selection in cloud computing environment. CSSaaS consists of two parts: monitoring \& indexing and filtration \& recommendation. The monitoring \& indexing component consist of a benchmarking/monitoring service, an indexing service, and an indexed services database. The filtration \& recommendation component includes a requirement identification service, a filtration service, and a ranking/recommendation service. CSSaaS contains a multicriteria decision making approach named as Fuzzy Linear Best Worst Method (FLBWM) which works under fuzzy environment. FLBWM has been tested in two conditions: optimization of parameters and IaaS service selection. Results are obtained on sensitivity analysis, rank correlation, collaborative decision making. Obtained results demonstrate that CSSaaS performs better compared to other state of the art solutions. CSSaaS do not consider discrete values for evaluation of cloud services.

It can be observed from above discussion that service selection in cloud environment is a decision making problem which has been solved using MCDM techniques by researchers. Authors have used either subjective or objective attributes to provide Joyent to QoS criteria in MCDM techniques. There is scope to integrate both subjective and objective attributes for finding suitable service as per users' requirements. We have integrated both subjective and objective attributes' weight to search the cloud services based on users' QoS demands.

\section{Brokering model for service selection (BSS)}

In this section, we describe our proposed broker based cloud service selection model (BSS) for cloud environment. BSS employs users' feedback of cloud services and third party given tested data for finding best service for a user. It consists of four modules: service selection, users' feedback, feedback aggregation service and benchmark tested data modules. The proposed model is shown in Fig. 1. Users interact to broker to find suitable service as per their needs. Users provide functional and non-functional requirements as QoS demands as well as preference to these QoS parameters in linguistic form. The broker return service or set of services based on given search.

- Service Selection Module: This module receives requests from users with functional and non-functional QoS parameters. Users also provide 


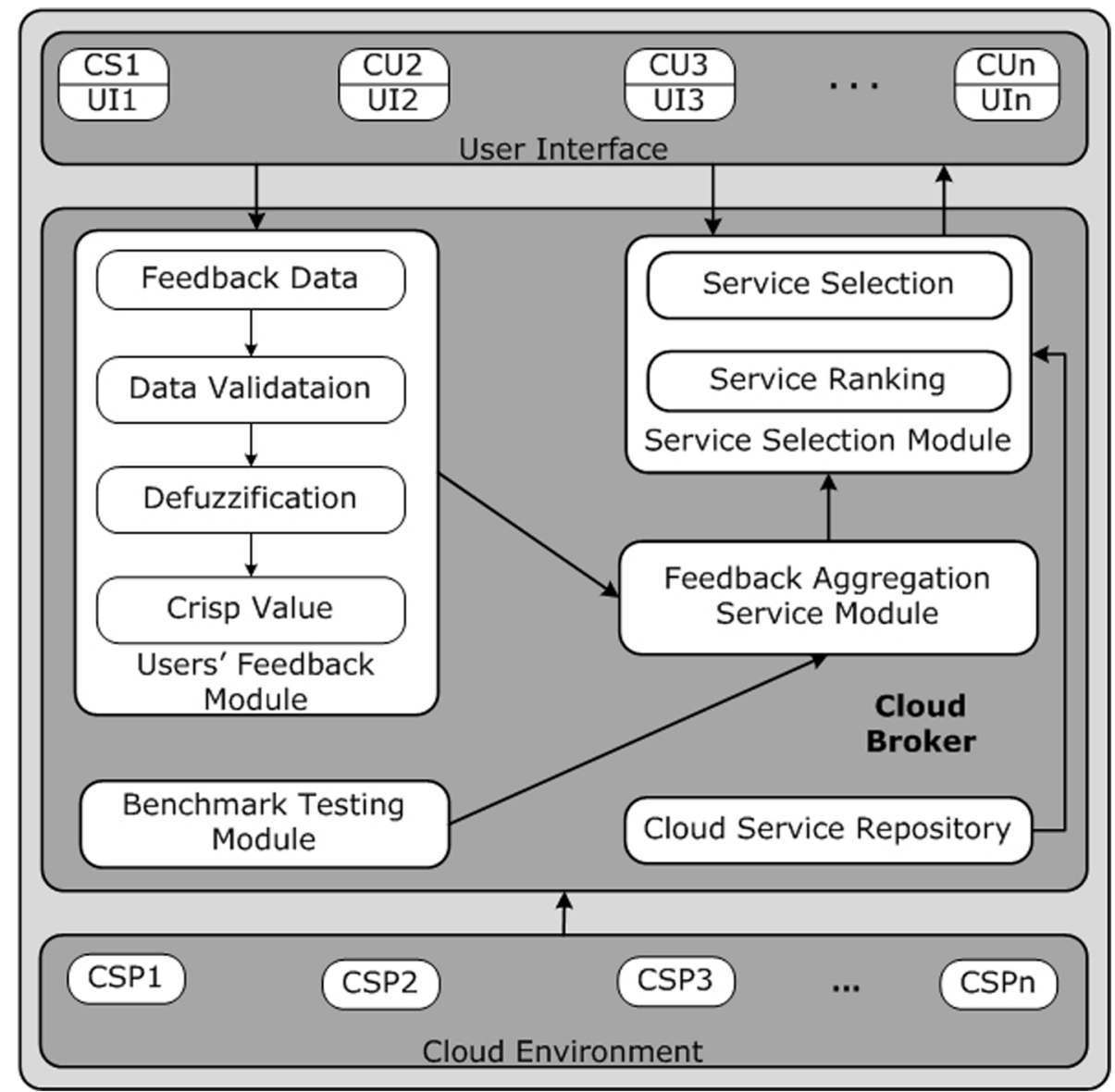

Fig. 1 Cloud Service Selection Model

preference to these parameters in linguistic form. This module performs initial processing whether the service can be accepted or not. This module also sends requests to other module to get required data of a cloud service. This module gathers list of services from cloud service repository which stores presently available services. From this list of service, this module finds which services can fulfill subjective and objective requirements of the user. It gathers aggregated feedback of selected list of cloud services. Based on gathered data, this module ranks these cloud services. The user is provided this list of services with their rank.

- Users' Feedback Module: This module is responsible to collect and manage feedback given by users who have used cloud services. This module is responsible for collecting and validating QoS data of services. It is very important to collect correct QoS data. End Users are main source of getting QoS data but due to competitive environment they may provide overestimated data of cloud services. This may lead to false decision in service selection. Users provide their subjective assessment about a QoS parameter of a cloud service which is generally in linguistic term such as high, good, bad [43]. This assessment can be considered as subjective attribute of QoS criteria. Feedback is hard to understand as it is vague in nature and difficult to quantify. Fuzzy logic can help into understanding and conversion of feedback values into crisp values. The module uses rating given in Table 1 to convert linguistic values into fuzzy numbers. Triangular fuzzy number [44] is used to convert it. The module contains defuzzification process to find crisp value from these fuzzy ratings. These QoS criteria are based on Service Measurement Index (SMI) [14].

- Benchmark Testing Module: Trusted third parties are employed to find common performance statistics of a cloud service. They employ various testing scenario as a benchmark to obtain performance of cloud services in various metrics such as availability, response time, reliability, etc. The benchmark test can be divided into two: common test and specific test. Common tests are those which are executed on 
Table 1 User Feedback Scale with Fuzzy Ratings

\begin{tabular}{lll}
\hline \multicolumn{1}{c}{ User Feedback for } & & Fuzzy Ratings \\
\hline Benefit Attribute & Cost Attribute & \\
\hline Worst & Best & $(1,1,1)$ \\
Bad & Very Good & $(1,3,5)$ \\
Good & Good & $(3,5,7)$ \\
Very Good & Bad & $(5,7,9)$ \\
Best & Worst & $(7,9,9)$ \\
\hline
\end{tabular}

every cloud service to measure QoS attributes. Specific tests are those which are performed as per needs of a user such as high computation tests, speed test for encryption, etc. These tests can be considered to obtain objective attributes of a cloud service. Objective attribute values are represented into quantified format such as availability as $99.9 \%$, response time as $100 \mathrm{~ms}$, etc. Benchmark testing is performed in different scenario and many times to find performance of cloud services. CloudHarmony and CloudSpectator are well known benchmark test providers. This module stores objective attribute values of cloud services for further use.

- Feedback Aggregation Service Module: This module performs aggregation of subjective and objective feedback of a cloud service. Neither subjective nor objective feedback of a cloud service is sufficient to describe its functionality. Users' feedback module provides subjective feedback while benchmark testing module provides objective feedback of a cloud service. User provides an preference to a QoS attributes of a cloud service such as high reliable cloud service. This preference is converted into a weight value in computation. As the preference is given into linguistic form, fuzzy rating as given in 1 is employed to compute its crisp value. The preference to subjective and objective attributes can be different. This lets to know the user that which attributes are good for requested service.

- Cloud Service Repository: This module is the catalog for storing information of cloud services and QoS parameters given by service providers. It is having information related to the cloud services which are offered by different cloud service providers. The past information of cloud services is stored with this module. Service ranking and selection module access information of cloud services form this module for ranking them.

\section{BSS technique}

In this section, we describe out proposed method for service selection in cloud environment. The method employs aggregated feedback of subjective and objective attributes of cloud services. In our model as shown in previous section, there is only one trusted third party which provides values of objective attributes and sufficient users have provided subjective attribute feedback of cloud services. The trusted third party provides single set of objective attributes as asked by cloud broker for set of cloud services. The service selection process is shown in algorithm (1). The detailed description of BSS method as follows: The decision matrix, D is shown below which is having m cloud services and $\mathrm{n}$ QoS criteria:

$$
D=\left[\begin{array}{ccccc}
r_{11} & r_{12} & r_{13} & \ldots & r_{1 n} \\
r_{21} & r_{22} & r_{23} & \ldots & r_{2 n} \\
\vdots & \vdots & \vdots & \vdots & \vdots \\
r_{m 1} & r_{m 2} & r_{m 3} & \ldots & r_{m n}
\end{array}\right]
$$

The decision matrix consists of cloud services form different cloud providers hence it carries different scales for QoS attributes. It must be normalized in order to apply common method for evaluation. The benefit QoS attributes are normalized using Eq. (1).

$$
x_{i j}=\frac{r_{i j}-r_{\min }}{r_{\max }-r_{\min }}
$$

The cost QoS attributes are normalized using following Eq. (2).

$$
x_{i j}=\frac{r_{\max }-r_{i j}}{r_{\max }-r_{\min }}
$$

In the next step, weighted normalized decision matrix is computed. The weight is aggregated of subjective and objective weights. The subjective weight is computed on the values derived from feedback of users. The subjective feedback values are in linguistic form. The feedback on different QoS parameters is collected as per terms given in Table 1 for both benefit and cost QoS attributes. A decision matrix with available service alternatives and criteria feedback is formed as shown below.

$$
D=\left[\begin{array}{ccccc}
a_{11} & a_{12} & a_{13} & \ldots & a_{1 n} \\
a_{21} & a_{22} & a_{23} & \ldots & a_{2 n} \\
\vdots & \vdots & \vdots & \vdots & \vdots \\
a_{m 1} & a_{m 2} & a_{m 3} & \ldots & a_{m n}
\end{array}\right]
$$

Here, $a_{i j}$ represents feedback value of $i^{\text {th }}$ service alternative with respect to $j^{\text {th }}$ QoS criteria. Users always interested in one or other QoS attributes with some more values. This preference is assigned through a weight value. The preference parameters and their corresponding values are shown in Table 2. 


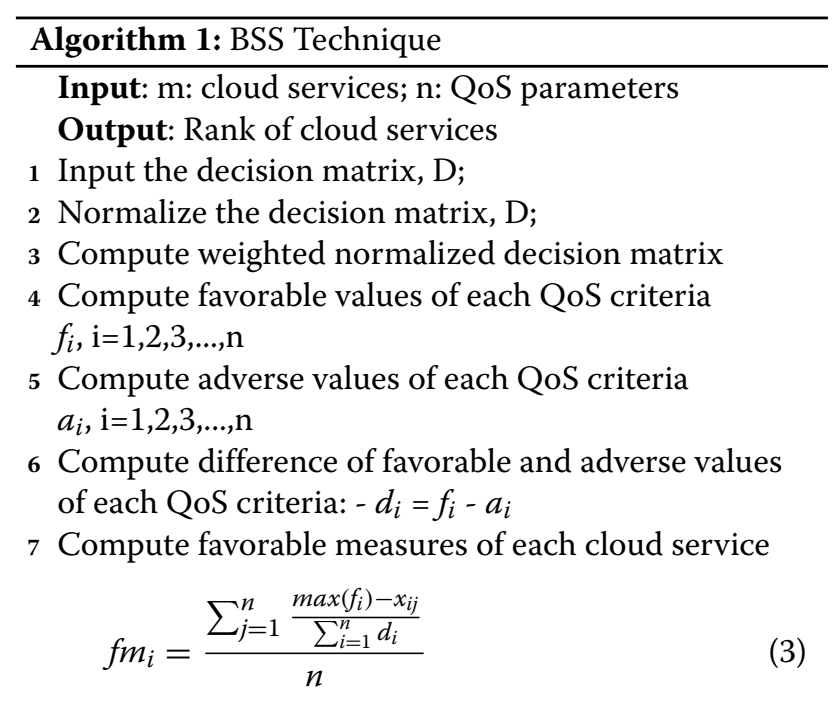

8 Compute adverse measures of each cloud service

$$
a m_{i}=\frac{\max \left(s_{i}\right)}{n}
$$

here,

$$
s_{i}=\frac{\max \left(f_{i}\right)-x_{i j}}{n \times \sum_{i=1}^{n} d_{i}}
$$

9 Compute total measure of each cloud service using equation

$$
t m_{i}=\frac{\frac{f m_{i}-\min \left(f m_{i}\right)}{\max \left(f m_{i}\right)-\min \left(f m_{i}\right)}+\frac{a m_{i}-\min \left(a m_{i}\right)}{\max \left(a m_{i}\right)-\min \left(a m_{i}\right)}}{2}
$$

10 Sort the values of total measure in ascending order 11 Lower the value of total measure, higher is the rank 12 Select the cloud service with highest rank;

These preference parameters are applied to respective QoS criteria using following equation.

$$
\tilde{r_{i j}}=\tilde{p_{j}} \otimes \tilde{a_{i j}}, \quad j=1,2,3, \ldots, m ; \quad i=1,2,3, \ldots, n
$$

Here, $\tilde{p}_{j}$ is the preference value assigned to QoS criteria $j$ and $a_{i j}$ is the feedback value on $j^{\text {th }}$ QoS criteria of $i^{\text {th }}$ cloud service. The obtained decision matrix after multiplying preference parameters is in fuzzy form. Defuzzification is required to compute numerical crisp values from them.

Table 2 Preference Parameters with Fuzzy Ratings

\begin{tabular}{ll}
\hline Preference Parameters & Fuzzy Ratings \\
\hline Not Important & $(1,1,1)$ \\
Important & $(1,3,5)$ \\
More Important & $(3,5,7)$ \\
Strongly Important & $(5,7,9)$ \\
Absolutely Important & $(7,9,9)$
\end{tabular}

Table 3 Decision Matrix for Subjective Weight

\begin{tabular}{llllll}
\hline $\begin{array}{l}\text { Cloud } \\
\text { Services }\end{array}$ & SQoS1 & SQoS2 & SQoS3 & SQoS4 & SQoS5 \\
\hline CS1 & Good & Good & Good & Very Good & Good \\
CS2 & Best & Worst & Best & Best & Bad \\
CS3 & Very Good & Very Good & Bad & Very Good & Very Good \\
CS4 & Worst & Good & Best & Very Good & Very Good \\
CS5 & Very Good & Good & Very Good & Good & Good \\
CS6 & Good & Good & Good & Good & Good \\
CS7 & Good & Very Good & Good & Good & Worst \\
CS8 & Bad & Very Good & Best & Best & Worst \\
CS9 & Worst & Good & Best & Very Good & Good \\
CS10 & Worst & Very Good & Very Good & Good & Best \\
\hline
\end{tabular}

Beta distribution [45] is employed to compute numerical crisp value from a triangular fuzzy number.

$$
a=\frac{l+m+u}{3}
$$

Here, $1, \mathrm{~m}$ and $\mathrm{u}$ denotes left hand, middle and right hand number of a triangular fuzzy number, respectively. Normalization of criteria values is required so that they can be compared with other values. The QoS criteria for a cloud service can be categorized into two: benefit and cost. The benefit and cost QoS attributes are normalized using Eqs. (1) and (2), respectively.

The subjective weight of QoS attributes is calculated with following equation:

$$
s w_{i}=\frac{\sum_{i=1}^{m} x_{i j}}{\sum_{i=1}^{m} \sum_{j=1}^{n} x_{i j}}
$$

Benchmark testing data provides real time values of QoS attributes of a cloud service. They are more important and can be considered error-free. Objective weight of a QoS criteria is computed through benchmark tested data. Entropy method is employed for objective weight

Table 4 Decision Matrix with Fuzzy Ratings

\begin{tabular}{llllll}
\hline Cloud Services & SQoS1 & SQoS2 & SQoS3 & SQoS4 & SQoS5 \\
\hline CS1 & $(3,5,7)$ & $(3,5,7)$ & $(3,5,7)$ & $(5,7,9)$ & $(3,5,7)$ \\
CS2 & $(7,9,9)$ & $(7,9,9)$ & $(7,9,9)$ & $(7,9,9)$ & $(5,7,9)$ \\
CS3 & $(5,7,9)$ & $(1,3,5)$ & $(1,3,5)$ & $(5,7,9)$ & $(1,3,5)$ \\
CS4 & $(1,1,1)$ & $(3,5,7)$ & $(7,9,9)$ & $(5,7,9)$ & $(1,3,5)$ \\
CS5 & $(5,7,9)$ & $(3,5,7)$ & $(5,7,9)$ & $(3,5,7)$ & $(3,5,7)$ \\
CS6 & $(3,5,7)$ & $(3,5,7)$ & $(3,5,7)$ & $(3,5,7)$ & $(3,5,7)$ \\
CS7 & $(3,5,7)$ & $(1,3,5)$ & $(3,5,7)$ & $(3,5,7)$ & $(7,9,9)$ \\
CS8 & $(1,3,5)$ & $(1,3,5)$ & $(7,9,9)$ & $(7,9,9)$ & $(7,9,9)$ \\
CS9 & $(1,1,1)$ & $(3,5,7)$ & $(7,9,9)$ & $(5,7,9)$ & $(3,5,7)$ \\
CS10 & $(1,1,1)$ & $(1,3,5)$ & $(5,7,9)$ & $(3,5,7)$ & $(1,1,1)$ \\
\hline
\end{tabular}


Table 5 Decision Matrix with Numerical Crisp Values

\begin{tabular}{llllll}
\hline Cloud Services & SQoS1 & SQoS2 & SQoS3 & SQoS4 & SQoS5 \\
\hline CS1 & 5 & 5 & 5 & 7 & 5 \\
CS2 & 8.33 & 8.33 & 8.33 & 8.33 & 7 \\
CS3 & 7 & 3 & 3 & 7 & 3 \\
CS4 & 1 & 5 & 8.33 & 7 & 3 \\
CS5 & 7 & 5 & 7 & 5 & 5 \\
CS6 & 5 & 5 & 5 & 5 & 5 \\
CS7 & 5 & 3 & 5 & 5 & 8.33 \\
CS8 & 3 & 3 & 8.33 & 8.33 & 8.33 \\
CS9 & 1 & 5 & 8.33 & 7 & 5 \\
CS10 & 1 & 3 & 7 & 5 & 1 \\
\hline
\end{tabular}

calculation. Shannon has introduced entropy theory in information theory [46]. Entropy method is very simple to compute disorder degree between set of values. Here, QoS criteria of a cloud service is evaluated through objective values. Based on the value, weight of QoS criteria is computed. According to entropy method if the disorder degree is less the weight is high. Objective weight of a QoS attribute is computed using following equation:

$$
o w_{i}=\frac{1-E_{i}}{\sum_{i=1}^{n}\left(1-E_{i}\right)}
$$

$$
\text { Here }
$$

$$
\begin{aligned}
& \sum_{i=1}^{n} o w_{i}=1 \\
& E_{i}=-k \sum_{j=1}^{m} r_{i j} \ln r_{i j}, \quad j=1,2,3, \ldots, m \quad k=\frac{i}{\ln m}
\end{aligned}
$$

Total weight of a QoS attribute $i$ is computed using Eq. (14):

$$
w_{i}=\frac{s w_{i}+o w_{i}}{2}
$$

Table 6 Normalized Decision Matrix

\begin{tabular}{llllll}
\hline Cloud Services & SQoS1 & SQoS2 & SQoS3 & SQoS4 & SQoS5 \\
\hline CS1 & 0.545 & 0.624 & 0.375 & 0.600 & 0.454 \\
CS2 & 1 & 0 & 1 & 1 & 0.181 \\
CS3 & 0.818 & 1 & 0 & 0.600 & 0.727 \\
CS4 & 0 & 0.624 & 1 & 0.600 & 0.727 \\
CS5 & 0.818 & 0.624 & 0.750 & 0 & 0.454 \\
CS6 & 0.545 & 0.624 & 0.375 & 0 & 0.454 \\
CS7 & 0.545 & 1 & 0.375 & 0 & 0 \\
CS8 & 0.272 & 1 & 1 & 1 & 0 \\
CS9 & 0 & 0.624 & 1 & 0.600 & 0.454 \\
CS10 & 0 & 1 & 0.750 & 0 & 1 \\
\hline
\end{tabular}

Table 7 Subjective Weight of QoS Criteria

\begin{tabular}{ll}
\hline Subjective QoS Criteria & Subjective Weight \\
\hline SQoS1 & 0.168 \\
SQOS2 & 0.262 \\
SQoS3 & 0.242 \\
SQoS4 & 0.162 \\
SQoS5 & 0.164 \\
\hline
\end{tabular}

In the next step, favorable and adverse values of each QoS criteria is computed. Favorable values are those which provides positive measures and adverse values are those which provides negative measures. Favorable value of cloud service alternatives for a QoS attribute is computed as: $\left\{f_{j}=\max x_{i j}, \mathrm{j}=1,2,3, \ldots, \mathrm{m}\right.$. $\}$ Adverse values of cloud service alternatives for a QoS attribute is computed as: $\left\{a_{j}=\min x_{i j}, \mathrm{j}=1,2,3, \ldots, \mathrm{m}\right.$. $\}$ In next step, the difference of favorable and adverse values of each service alternative is computed.

$$
d_{j}=f_{j}-a_{j}, \quad j=1,2,3, \ldots, m
$$

The next step comprises of computation of favorable measure of each cloud service alternative.

$$
f m_{i}=\frac{\sum_{j=1}^{n} \frac{\max \left(f_{i}\right)-x_{i j}}{\sum_{i=1}^{n} d_{i}}}{n}
$$

The adverse measure of each cloud service alternative is computed with following equation:

$$
a m_{i}=\frac{\max \left(s_{i}\right)}{n}
$$

here,

$$
s_{i}=\frac{\max \left(f_{i}\right)-x_{i j}}{n \times \sum_{i=1}^{n} d_{i}}
$$

Table 8 Decision Matrix for Objective Weight

\begin{tabular}{llllll}
\hline Cloud Services & OQoS1 & OQoS2 & OQoS3 & OQoS4 & OQoS5 \\
\hline CS1 & 63.44 & 66 & 56.82 & 91 & 0.17 \\
CS2 & 111.95 & 119.63 & 100.5 & 131.81 & 0.42 \\
CS3 & 77.48 & 23.43 & 40.23 & 80.67 & 0.12 \\
CS4 & 5.45 & 78.56 & 109.2 & 84.2 & 0.12 \\
CS5 & 82.2 & 67.97 & 78.49 & 61.8 & 0.24 \\
CS6 & 41.85 & 70.29 & 63.1 & 63.44 & 0.24 \\
CS7 & 58.42 & 31.22 & 68.45 & 78.15 & 1.05 \\
CS8 & 37.05 & 36.15 & 102.74 & 132.87 & 1.67 \\
CS9 & 42.05 & 59.63 & 174.5 & 97.16 & 0.21 \\
CS10 & 5.38 & 47.17 & 84.56 & 81.23 & 0.056 \\
\hline
\end{tabular}


In final step total measure is computed with following equation:

$$
t m_{i}=\frac{\frac{f m_{i}-\min \left(f m_{i}\right)}{\max \left(f m_{i}\right)-\min \left(f m_{i}\right)}+\frac{a m_{i}-\min \left(a m_{i}\right)}{\max \left(a m_{i}\right)-\min \left(a m_{i}\right)}}{2}
$$

The total measure values are sorted in ascending order. Lower value is assigned higher rank. Cloud service with highest rank is assigned to user.

\section{Experiment and result}

The proposed brokering model, BSS for service selection has been simulated using CloudSim [47], simulation toolkit widely used for simulation of cloud computing environment. BSS in evaluated through a case study which demonstrate effectiveness and usefulness in cloud service selection. The dummy dataset is used to compute subjective weight while a real dataset which has been collected by kumar et al. [35] is used to compute objective weight of QoS parameters. The dataset is created from benchmark service provider CloudHarmony. BSS divides service selection in two parts: aggregate weight computation and service ranking.

\section{Weight computation}

The subjective weight of QoS criteria is computed on the basis of feedback provided by users which is in linguistic terms. The service alternatives Joyent, Amazon, CityCloud, Softlayer, HP, Century Link, Rackspace, Google, Linode and Azure which are further represented as CS1, CS2, CS3, CS4, CS5, CS6, CS7, CS8, CS9 and CS10, respectively as given in paper ([35]) are considered. The QoS criteria CPU performance, Disk I/O consistency, Disk performance, Memory performance, and Cost which are further referred as SQoS1, SQoS2, SQoS3, SQoS4, and SQoS5, respectively are considered for evaluation of cloud services. Disk performance and memory performance are considered as benefit and others as cost attributes.

Table 9 Normalized Decision Matrix

\begin{tabular}{llllll}
\hline Cloud Services & OQoS1 & OQoS2 & OQoS3 & OQoS4 & OQoS5 \\
\hline CS1 & 0.544 & 0.557 & 0.123 & 0.410 & 0.929 \\
CS2 & 1 & 0 & 0.448 & 0.985 & 0.774 \\
CS3 & 0.676 & 1 & 0 & 0.265 & 0.960 \\
CS4 & 0.0006 & 0.426 & 0.513 & 0.315 & 0.960 \\
CS5 & 0.720 & 0.537 & 0.284 & 0 & 0.885 \\
CS6 & 0.342 & 0.512 & 0.170 & 0.023 & 0.885 \\
CS7 & 0.497 & 0.919 & 0.210 & 0.230 & 0.384 \\
CS8 & 0.297 & 0.867 & 0.465 & 1 & 0 \\
CS9 & 0.344 & 0.623 & 1 & 0.497 & 0.904 \\
CS10 & 0 & 0.753 & 0.330 & 0.273 & 1
\end{tabular}

Table 10 Objective Weight of QoS Criteria

\begin{tabular}{ll}
\hline Objective QoS Criteria & Objective Weight \\
\hline OQOS1 & 0.018 \\
OQOS2 & 0.139 \\
OQOS3 & 0.014 \\
OQoS4 & 0.050 \\
OQOS5 & 0.779 \\
\hline
\end{tabular}

The linguistic terms given in Table 1 are used to describe the feedback values on different QoS criteria by a user. The decision matrix shown in Table 3 for obtaining subjective weights is considered.

The linguistic terms are converted into fuzzy ratings as per given in Table 1 values, which is shown in Table 4. The defuzzification is applied to convert fuzzy ratings into numerical crisp values, which is shown in Table 5. These values are further normalized to make them in one form and shown in Table 6. The subjective weight of QoS criteria is obtained and shown in Table 7.

The dataset which has been generated through benchmark testing, given in paper ([35]) has been used to compute objective weight of QoS criteria. The decision matrix computed from the dataset is shown in Table 8.

Normalized decision matrix for objective weight computation is shown in Table 9. The objective weights obtained from normalized decision matrix is shown in Table 10. Total weight of required QoS attributes is computed using Eq. (14) and shown in Table 11.

\section{Service ranking}

In next step after computing integrated weight of all QoS parameters, ranking of cloud services is performed. Service ranking method with numerical calculation is also shown for better understanding of working of proposed BSS method.

- Construct Normalized Decision Matrix: The decision matrix shown in Table 8 is employed to evaluate BSS method. The normalized matrix which is shown in Table 9 is computed using Eqs. (1) and (2) for benefit and cost criteria, respectively.

Table 11 Total Weight of QoS Criteria

\begin{tabular}{ll}
\hline QoS Criteria & Total Weight \\
\hline QoS1 & 0.093 \\
QoS2 & 0.201 \\
QoS3 & 0.128 \\
QoS4 & 0.106 \\
QoS5 & 0.472 \\
\hline
\end{tabular}


Table 12 Normalized Decision Matrix

\begin{tabular}{llllll}
\hline Cloud Services & OC1 & OC2 & OC3 & OC4 & OC5 \\
\hline CS1 & 0.091 & 0.146 & 0.029 & 0.066 & 0.152 \\
CS2 & 0.168 & 0 & 0.108 & 0.159 & 0.127 \\
CS3 & 0.114 & 0.262 & 0 & 0.043 & 0.157 \\
CS4 & 0.0001 & 0.112 & 0.124 & 0.051 & 0.157 \\
CS5 & 0.121 & 0.141 & 0.068 & 0 & 0.145 \\
CS6 & 0.057 & 0.134 & 0.041 & 0.004 & 0.145 \\
CS7 & 0.083 & 0.241 & 0.051 & 0.037 & 0.063 \\
CS8 & 0.049 & 0.227 & 0.113 & 0.162 & 0 \\
CS9 & 0.058 & 0.163 & 0.242 & 0.081 & 0.148 \\
CS10 & 0 & 0.197 & 0.079 & 0.044 & 0.164 \\
\hline
\end{tabular}

- Construct Weighted Normalized Decision Matrix: The decision matrix shown in (9) is multiplied with the weight values as shown in Table 11 to construct the weighted normalized decision matrix. The matrix is shown in Table 12.

- Compute Favorable and Adverse Values: The favorable and adverse value of each QoS criteria with respect to each cloud service is computed and denoted as $f_{i}$ and $a_{i}$, respectively. Favorable values are the maximum and adverse values are minimum of individual QoS criteria.

- Compute Favorable and Adverse Measure: Favorable, fm and Adverse am measures of each cloud service are computed using Eqs. (16) and (17), respectively.

- Compute Total Measure (tm): The total measure (tm) is computed using Eq. 19. The obtained values are shown in the Table 13.

- Assign Rank: Rank to cloud services are assigned on the basis of total measure, $t m$ as shown in Table 14. It can be observed from the Table 14 that Amazon is ranked first and Linode is ranked as second. The order of rank is: Amazon, Linode, HP, Softlayer,

Table 13 Total Measure

\begin{tabular}{ll}
\hline Cloud Services & Total Measure $(\mathbf{t m})$ \\
\hline CS1 & 0.749 \\
CS2 & 0.0 \\
CS3 & 1.0 \\
CS4 & 0.594 \\
CS5 & 0.426 \\
CS6 & 0.731 \\
CS7 & 0.885 \\
CS8 & 0.613 \\
CS9 & 0.349 \\
CS10 & 0.763 \\
\hline
\end{tabular}

Table 14 Rank of Cloud Services

\begin{tabular}{ll}
\hline Cloud Services & Rank \\
\hline CS1 (Joyent) & 7 \\
CS2 (Amazon) & 1 \\
CS3 (City-Cloud) & 10 \\
CS4 (Softlayer) & 4 \\
CS5 (HP) & 3 \\
CS6 (Century Link) & 6 \\
CS7 (Rackspace) & 9 \\
CS8 (Google) & 5 \\
CS9 (Linode) & 2 \\
CS10 (Azure) & 8 \\
\hline
\end{tabular}

Google, Century Link, Joyent, Rackspace, Azure and City-Cloud.

\section{Sensitivity analysis}

The sensitivity analysis is performed to check the robustness of proposed model in different scenario. It is also performed to evaluate the proposed model for rank reversal problem. If ranking model gives non-optimal ranks in case of addition and removal of cloud services then the model cannot solve rank reversal problem. The proposed model is also verified for rank reversal problem through sensitivity analysis. Two scenario is possible: first some cloud services are removed and another some cloud services as added in existing cloud services set.

Five cloud services, Joyent, Amazon, City-Cloud, Softlayer and HP are taken for first case scenario. The sensitivity analysis is performed by removing City-Cloud cloud service in second experiment. In the first experiment all cloud services were considered. In third experiment, HP cloud service were removed. The total measure values are computed and shown in Table 15. The rank of cloud services are shown in Fig. 2. It can be deduced from above two results that the rank of cloud services are consistent. Hence, BSS model is robust in scenario where cloud services are removed in experiments.

Table 15 Total Measure for Each Experiment

\begin{tabular}{llll}
\hline Cloud Services & \multicolumn{2}{l}{ Experiment } & \\
\cline { 2 - 4 } & Exp1-tm & Exp2-tm & Exp3-tm \\
\hline Joyent & 0.49 & 0.316 & 0.822 \\
Amazon & 0.0 & 0.059 & 0.0 \\
City-Cloud & 1.0 & - & 0.879 \\
Softlayer & 0.929 & 1.0 & 0.686 \\
HP & 0.238 & 0.22 & - \\
\hline
\end{tabular}




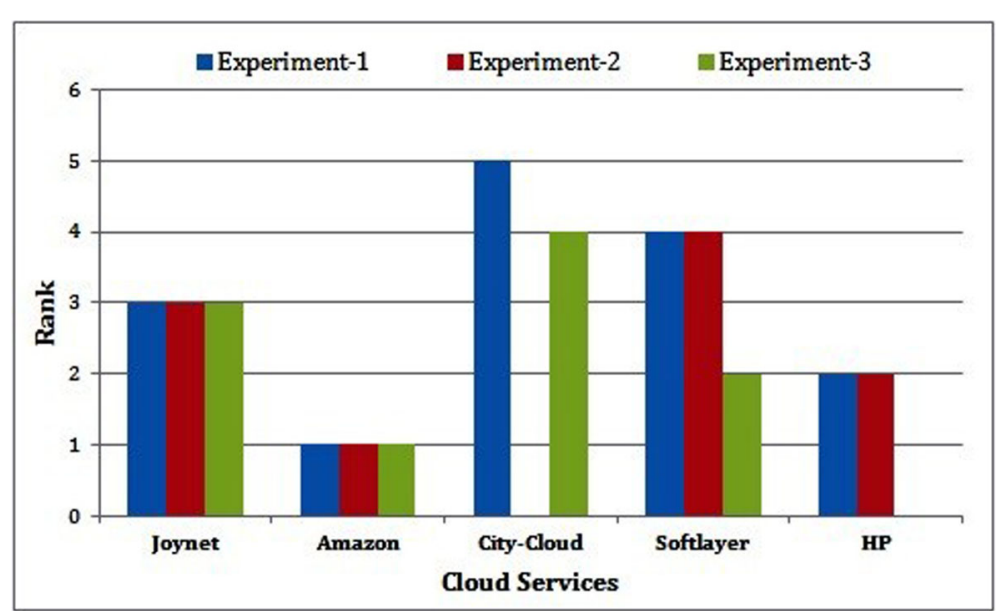

Fig. 2 Rank for Case Scenario 1

The sensitivity analysis for the second case scenario is performed starting with five cloud services: Joyent, Amazon, City-Cloud, Softlayer and HP. In subsequent experiments, three more services are added. In second experiment Google, in third Google and Rackspace, in forth Google, Rackspace and Linode is added. The total measure and rank of all the cloud services in each experiment is computed. The total measure is shown in Table 16 and rank is shown in Fig. 3. It can be observed from the Table 16 that total measure of cloud services is consistent in each experiment until a better cloud service is not introduced. The results show that the BSS model is also consistent and robust in service addition scenario for rank reversal problem.

\section{Result validation}

The proposed model, BSS has been simulated and obtained results are compared with existing literature, AHP [26], improved TOPSIS [28] (I-TOPSIS), fuzzy TOPSIS [35] (F-TOPSIS) based frameworks. The obtained rank for each method is shown in Fig. 4. It can be observed that BSS model gives similar results as given by

Table 16 Total Measure for Each Experiment

\begin{tabular}{llll}
\hline \multirow{2}{*}{ Cloud Services } & \multicolumn{2}{l}{ Experiment } & \\
\cline { 2 - 4 } & Exp1-tm & Exp2-tm & Exp3-tm \\
\hline Joyent & 0.463 & 0.463 & 0.667 \\
Amazon & 0.0 & 0.0 & 0.0 \\
City-Cloud & 0.968 & 0.968 & 1.0 \\
Softlayer & 0.496 & 0.496 & 0.491 \\
HP & 0.484 & 0.484 & 0.506 \\
Google & 0.558 & 0.558 & 0.552 \\
Rackspace & - & 0.804 & 0.819 \\
Linode & - & - & 0.324 \\
\hline
\end{tabular}

I-TOPSIS and F-TOPSIS methods. It can be deduced from results that proposed framework is consistent for service selection.

BSS is also tested on execution time performance metric. User feedback on 500 cloud services in linguistic terms has been generated and a decision matrix is formed. In the first experiment 100 cloud services are evaluated for execution time. In subsequent experiments every time 100 cloud services are added and execution time is noted. The results are compared with AHP, I-TOPSIS and F-TOPSIS methods. BSS is more efficient in each experiments as shown in Fig. 5.

\section{Conclusion}

Multiple cloud providers aggregate services and forms a huge heterogeneous environment. These services possess similar characteristics and different performance levels which make service management a difficult task. Brokering model for service selection (BSS) is proposed for cloud environment. Service selection has been done by employing integrated weighting approach. Subjective and objective weights are combined to obtain integrated total weight of QoS parameters. Simulation based evaluation of service selection method with a case study has been done. Results are analyzed using sensitivity analysis to validate the rank reversal problem. BSS works similar to existing methods for cloud service ranking. Results obtained of execution time metric outperforms than other state-of-the art solutions. Overall, BSS is robust for ranking and selection of services in cloud environment.

In the future work, BSS can be extended for group decision making in cloud service ranking and selection. Hierarchical structure of QoS criteria can be employed to test its significance in weight computation and service selection. 


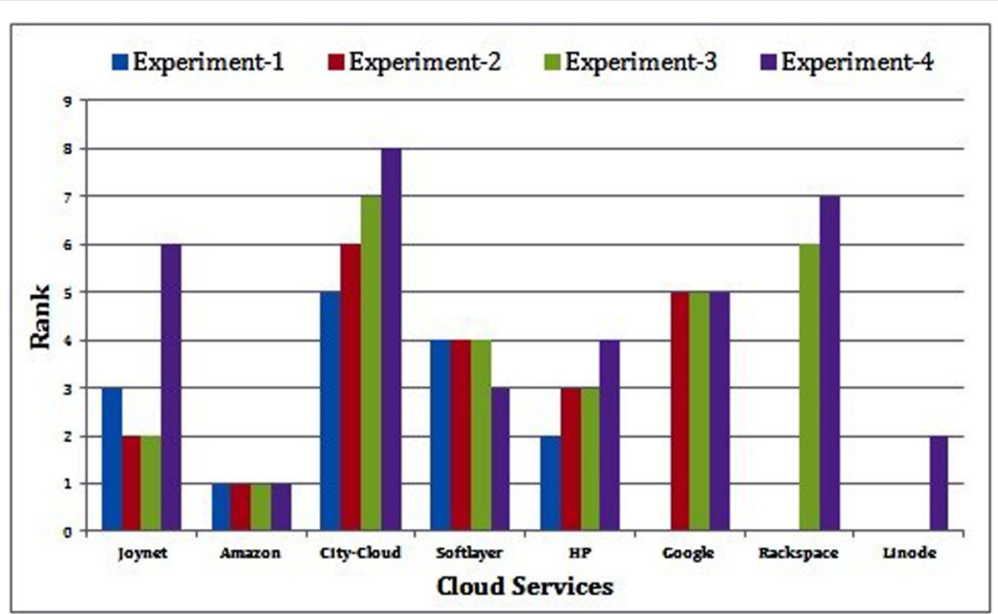

Fig. 3 Rank for Case Scenario 2

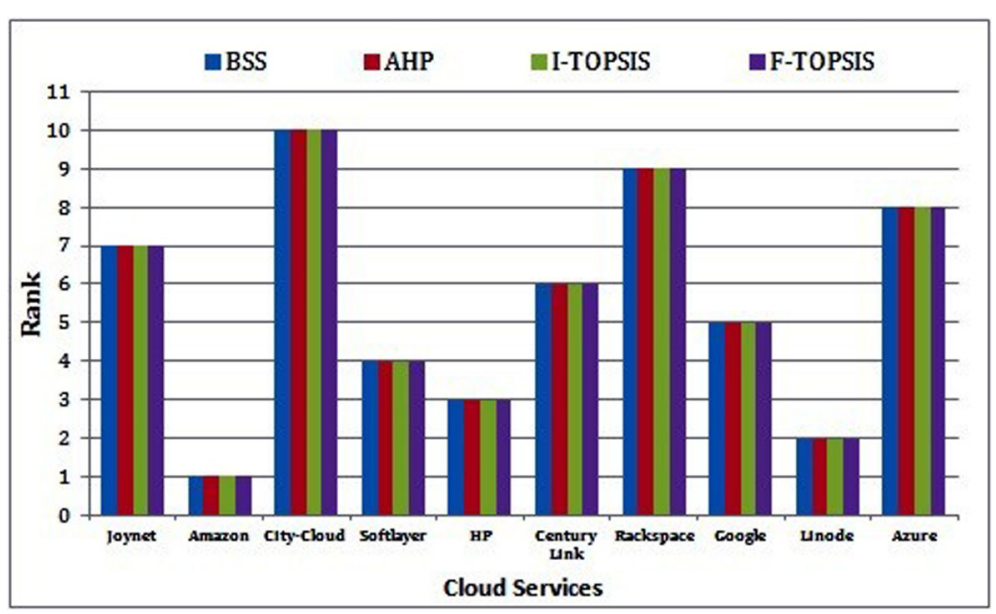

Fig. 4 Rank Analysis of All Cloud Services

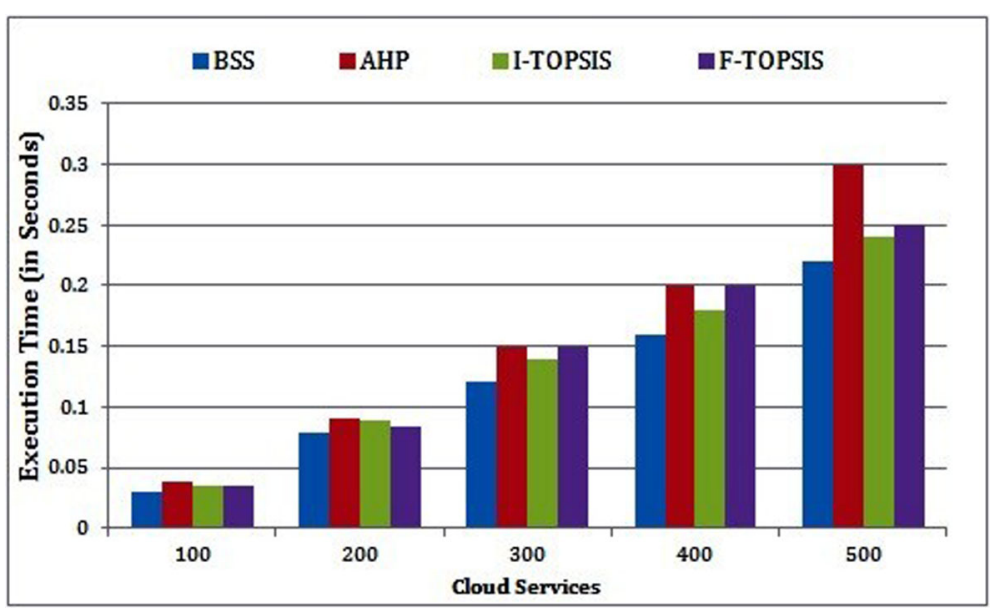

Fig. 5 Execution Time Analysis 


\section{Acknowledgments}

We sincerely thank the reviewers and the Editor for their valuable suggestions.

\section{Authors' contributions}

Sameer Singh Chauhan and Emmanuel S Pilli designed the study. Sameer Singh Chauhan performed simulation and write the paper. All authors reviewed and edited the manuscript. All authors read and approved the final manuscript.

\section{Funding}

No funds have been received from any agency for this research.

\section{Availability of data and materials}

The data used to support the finding of this study are available from the corresponding author upon request.

\section{Declarations}

\section{Competing interests}

The authors declare that they have no competing interests.

\section{Author details}

${ }^{1}$ Department of Computer Science and Engineering, Malaviya National Institute of Technology Jaipur, Rajasthan, India. ${ }^{2}$ Chancellor, Graphic Era Deemed to be University, Dehradun, India.

\section{Received: 9 November 2020 Accepted: 22 February 2021}

Published online: 14 April 2021

\section{References}

1. Buyya R, Yeo CS, Venugopal S, Broberg J, Brandic I (2009) Cloud computing and emerging it platforms: Vision, hype, and reality for delivering computing as the 5 th utility. Futur Gener Comput Syst 25(6):599-616. https://doi.org/10.1016/j.future.2008.12.001

2. Gill SS, Buyya R (2018) A taxonomy and future directions for sustainable cloud computing: 360 degree view. ACM Comput Surv (CSUR) 51(5):1-33. https://doi.org/10.1145/3241038

3. Hajjat M, Sun X, Sung Y-WE, Maltz D, Rao S, Sripanidkulchai K, Tawarmalani $M(2011)$ Cloudward bound: planning for beneficial migration of enterprise applications to the cloud. ACM SIGCOMM Comput Commun Rev 41(4):243-254. https://doi.org/10.1145/1851182.1851212

4. Jatoth C, Gangadharan G, Fiore U, Buyya R (2019) Selcloud: a hybrid multi-criteria decision-making model for selection of cloud services. Soft Comput 23(13):4701-4715. https://doi.org/10.1007/s00500-018-3120-2

5. Varia J (2011) Best practices in architecting cloud applications in the aws cloud. Cloud Comput Princ Paradigms 1:457-490

6. Gavvala SK, Jatoth C, Gangadharan G, Buyya R (2019) Qos-aware cloud service composition using eagle strategy. Futur Gener Comput Syst 90:273-290. https://doi.org/10.1016/j.future.2018.07.062

7. Kurze T, Klems M, Bermbach D, Lenk A, Tai S, Kunze M (2011) Cloud federation. Cloud Computing 1971548541:32-38

8. Buyya R, Srirama SN, Casale G, Calheiros R, Simmhan Y, Varghese B, Gelenbe E, Javadi B, Vaquero LM, Netto MA, et al (2018) A manifesto for future generation cloud computing: Research directions for the next decade. ACM Comput Surv (CSUR) 51(5):1-38. https://doi.org/10.1145/ 3241737

9. Kogias DG, Xevgenis MG, Patrikakis CZ (2016) Cloud federation and the evolution of cloud computing. Computer 49(11):96-99. https://doi.org/ 10.1109/MC.2016.344

10. Liaqat M, Chang V, Gani A, Hamid SHA, Toseef M, Shoaib U, Ali RL (2017) Federated cloud resource management: Review and discussion. J Netw Comput Appl 77:87-105. https://doi.org/10.1016/j.jnca.2016.10.008

11. Mell P, Grance T (2011) The nist definition of cloud computing. https:// doi.org/10.6028/NIST.SP.800-145

12. Santoso GZ, Jung Y, Seok S, Carlini E, Dazzi P, Altmann J, Violos J, Marshall $J$ (2017) Dynamic resource selection in cloud service broker. In: International Conference on High Performance Computing Simulation (HPCS). pp 233-235. https://doi.org/10.1109/HPCS.2017.43
13. Binnig C, Kossmann D, Kraska T, Loesing S (2009) How is the weather tomorrow?: towards a benchmark for the cloud. In: Proceedings of the Second International Workshop on Testing Database Systems, New York. pp 1-6. https://doi.org/10.1145/1594156.1594168

14. CSMIC (2014) Service measurement index (smi) framework version 2.1. Carnegie Mellon University Silicon Valley Moffett Field, CA, USA

15. Ma K, Bagula AB, Ajayi O (2019) Quality of service (qos) modelling in federated cloud computing. CoRR abs/1911.03051. http://arxiv.org/abs/ 1911.0305

16. Esposito C, Ficco M, Palmieri F, Castiglione A (2016) Smart cloud storage service selection based on fuzzy logic, theory of evidence and game theory. IEEE Trans Comput 65(8):2348-2362. https://doi.org/10.1109/TC. 2015.2389952

17. Masud AS, Ravindran AR, CRC Press AnimprintoftheTaylorandFrancisGroup (2008) Multiple criteria decision making. https://doi.org/10.1080/1331677X.2015.1075139

18. Eisa M, Younas M, Basu K, Awan I (2020) Modelling and simulation of qos-aware service selection in cloud computing. Simul Model Pract Theory 103:102108. https://doi.org/10.1016/j.simpat.2020.102108

19. Chen M-F, Tzeng G-H, Ding CG (2003) Fuzzy mcdm approach to select service provider. In: The 12th IEEE International Conference on Fuzzy Systems, St Louis. pp 572-577. https://doi.org/10.1109/FUZZ.2003. 1209427

20. Chen S-J, Hwang C-L (1992) Fuzzy multiple attribute decision making methods. In: Fuzzy Multiple Attribute Decision Making: Methods and Applications. Springer, Berlin. pp 289-486. https://doi.org/10.1007/978-3642-48318-9-3

21. Ma J, Fan Z-P, Huang L-H (1999) A subjective and objective integrated approach to determine attribute weights. Eur J Oper Res 112(2):397-404. https://doi.org/10.1016/S0377-2217(98)00141-6

22. Chauhan SS, Pilli ES, Joshi R, Singh G (2018) Upb: User preference based brokering for service ranking and selection in federated cloud. In: IEEE International Conference on Advanced Networks and Telecommunications Systems (ANTS). pp 1-6. https://doi.org/10.1109/ ANTS.2018.8710049

23. Patiniotakis I, Verginadis Y, Mentzas G (2015) Pulsar: preference-based cloud service selection for cloud service brokers. J Int Serv Appl 6(1):26. https://doi.org/10.1186/s13174-015-0042-4

24. Saaty RW (1987) The analytic hierarchy process what it is and how it is used. Math Modell 9(3):161-176. https://doi.org/10.1016/0270-0255-8790473-8

25. Chan KY, Kwong C, Dillon TS (2012) An enhanced fuzzy ahp method with extent analysis for determining importance of customer requirements. In: Computational Intelligence Techniques for New Product Design. Studies in Computational Intelligence, Berlin, Heidelberg. pp 79-93. https://doi. org/10.1007/978-3-642-27476-34

26. Garg SK, Versteeg S, Buyya R (2013) A framework for ranking of cloud computing services. Futur Gener Comput Syst 29(4):1012-1023. https:// doi.org/10.1016/j.future.2012.06.006. Special Section: Utility and Cloud Computing

27. Patiniotakis I, Rizou S, Verginadis Y, Mentzas G (2013) Managing imprecise criteria in cloud service ranking with a fuzzy multi-criteria decision making method. In: European Conference on Service-Oriented and Cloud Computing. pp 34-48. https://doi.org/10.1007/978-3-642-40651-54

28. Sidhu J, Singh S (2017) Improved topsis method based trust evaluation framework for determining trustworthiness of cloud service providers. J Grid Comput 15(1):81-105. https://doi.org/10.1007/s10723-016-9363-1

29. Saaty TL (2008) Decision making with the analytic hierarchy process. Int $J$ Serv Sci 1(1):83-98. https://doi.org/10.1504/IJSSCI.2008.017590

30. Hwang C-L, Yoon K (1981) Methods for multiple attribute decision making. In: Multiple Attribute Decision Making: Methods and Applications. Springer, Berlin. pp 58-191. https://doi.org/10.1007/978-3-642-48318-9-3

31. ur Rehman Z, Hussain FK, Hussain OK (2011) Towards multi-criteria cloud service selection. In: Fifth International Conference on Innovative Mobile and Internet Services in Ubiquitous Computing. pp 44-48. https://doi. org/10.1109/IMIS.2011.99

32. Anastasi GF, Carlini E, Coppola M, Dazzi P (2014) Qbrokage: A genetic approach for qos cloud brokering. In: IEEE 7th International Conference on Cloud Computing. pp 304-311. https://doi.org/10.1109/CLOUD.2014.49

33. Sundareswaran S, Squicciarini A, Lin D (2012) A brokerage-based approach for cloud service selection. In: IEEE Fifth International Conference on Cloud Computing. pp 558-565. https://doi.org/10.1109/CLOUD.2012.119 
34. Qu L, Wang Y, Orgun MA (2013) Cloud service selection based on the aggregation of user feedback and quantitative performance assessment. In: IEEE International Conference on Services Computing. pp 152-159. https://doi.org/10.1109/SCC.2013.92

35. Kumar RR, Mishra S, Kumar C (2018) A novel framework for cloud service evaluation and selection using hybrid mcdm methods. Arab J Sci Eng 43(12):7015-7030. https://doi.org/10.1007/s13369-017-2975-3

36. Paunovic M, Ralevic NM, Gajovic V, Mladenovic Vojinovic B, Milutinovic O (2018) Two-stage fuzzy logic model for cloud service supplier selection and evaluation. Math Probl Eng. https://doi.org/10.1155/2018/7283127

37. Alam KA, Ahmed R, Butt FS, Kim S-G, Ko K-M (2018) An uncertainty-aware integrated fuzzy ahp-waspas model to evaluate public cloud computing services. https://doi.org/10.1016/j.procs.2018.04.068

38. Chang D-Y (1996) Applications of the extent analysis method on fuzzy ahp. Eur J Oper Res 95(3):649-655. https://doi.org/10.1016/03772217(95)00300-2

39. Zavadskas EK, Turskis Z, Antucheviciene J, Zakarevicius A (2012) Optimization of weighted aggregated sum product assessment. Electron Electr Eng 122(6):3-6. https://doi.org/10.5755/j01.eee.122.6.1810

40. Tajvidi M, Ranjan R, Kolodziej J, Wang L (2014) Fuzzy cloud service selection framework. In: IEEE 3rd International Conference on Cloud Networking (CloudNet). pp 443-448. https://doi.org/10.1109/CloudNet. 2014.6969035

41. Tiwari RK, Kumar R (2021) G-topsis: a cloud service selection framework using gaussian topsis for rank reversal problem. J Supercomput 77:523-562. https://doi.org/10.1007/s11227-020-03284-0

42. Hussain A, Chun J, Khan M (2020) A novel framework towards viable cloud service selection as a service (cssaas) under a fuzzy environment. Futur Gener Comput Syst 104:74-91. https://doi.org/10.1016/j.future. 2019.09.043

43. Qu L, Wang Y, Orgun MA (2013) Cloud service selection based on the aggregation of user feedback and quantitative performance assessment. In: IEEE International Conference on Services Computing, Santa Clara. pp 152-159. https://doi.org/10.1109/SCC.2013.92

44. Shyama A. K. I., Pal M (2007) Triangular fuzzy matrices. Iran J Fuzzy Syst 4(1):75-87. https://doi.org/10.22111/ijfs.2007.359

45. Rahmani A, Hosseinzadeh Lotfi F, Rostamy-Malkhalifeh M, Allahviranloo T (2016) A new method for defuzzification and ranking of fuzzy numbers based on the statistical beta distribution. Adv Fuzzy Syst:1-8. https://doi. org/10.1155/2016/6945184

46. Shannon CE (2001) A mathematical theory of communication. ACM SIGMOBILE Mob Comput Commun Rev 5(1):3-55. https://doi.org/10. 1145/584091.584093

47. Calheiros RN, Ranjan R, Beloglazov A, De Rose CA, Buyya R (2011) Cloudsim: a toolkit for modeling and simulation of cloud computing environments and evaluation of resource provisioning algorithms. Softw Pract Exp 41(1):23-50. https://doi.org/10.1002/spe.995

\section{Publisher's Note}

Springer Nature remains neutral with regard to jurisdictional claims in published maps and institutional affiliations.

\section{Submit your manuscript to a SpringerOpen ${ }^{\circ}$ journal and benefit from:}

- Convenient online submission

- Rigorous peer review

- Open access: articles freely available online

- High visibility within the field

- Retaining the copyright to your article

Submit your next manuscript at $>$ springeropen.com 